\title{
Clinical image
}

\section{Umbilicus: A Site for Stoma in Hirschsprung's Disease}

\author{
Prince Raj, Yogesh Kumar Sarin* \\ Department of Pediatric Surgery, Maulana Azad Medical College, and associated Lok Nayak Hospital, New Delhi \\ This is an open-access article distributed under the terms of the Creative Commons Attribution License, which permits unrestricted use, \\ distribution, and reproduction in any medium, provided the original work is properly cited.
}

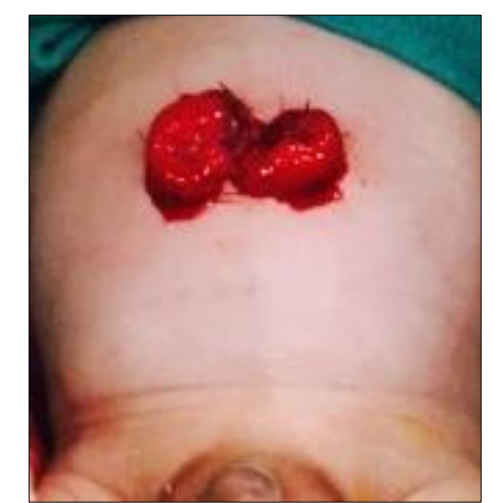

Figure 1: Umbilical divided colostomy

\begin{abstract}
A 13-day-old male baby with the symptoms suggestive of HD and barium enema showing the transition zone (TZ) at the recto-sigmoid was admitted with progressive abdominal distention not responding to rectal washes. The baby was planned for sigmoid colostomy and seromuscular biopsy. A transverse skin incision of $2 \mathrm{cms}$ was made through the umbilicus under general anesthesia. The skin, subcutaneous tissue, and fascia were incised, and the umbilical vessels and urachal remnant were individually ligated apart from the opening in the fascia. At this point, a urethral sound was passed per rectally to guide the sigmoid loop towards the umbilicus. Stay sutures were taken and the loop of sigmoid colon was taken out. Biopsies were taken from the recto-sigmoid junction and site proximal to TZ. The bowel wall was fixed separately to the peritoneum and fascia with interrupted 5-0 absorbable sutures. The bowel was opened and everted with suturing to the skin (Fig. 1).
\end{abstract}

Management of Hirschsprung's disease (HD) has evolved in last one decade from staged procedure to single stage trans-anal pull through. But in developing country like ours, the presentation is generally late (most of them brought with severely dilated colon or enterocolitis) and where there is lack of facility for frozen section biopsy, staged procedures are still the standard of care. In this era of minimal access surgery, where a lot of stress is laid on cosmesis, role of umbilical incision for fashioning of colostomy and taking biopsies is a good alternative. Though trans-umbilical colostomies have been occasionally done in past for HD and anorectal malformations 1,2,3, they are still not widely used.

This technique has several advantages:
1. Less time consuming, cost effective and easy to perform.

2. Provides easy access to both the sigmoid and transverse colon.

3. Umbilicus lies on the center of the abdomen and hence the stoma bag can be well placed and stoma care will be easy.

4. Better cosmesis as the scar will not be visible once the stoma is closed.

5. Does not require advanced surgical skill as in laparoscopy.

6. Delayed trans-anal pull through can still be performed through the same incision.

In today's world smitten with minimal accesses surgery, this approach will serve as an attractive alternative in the staged management of HD, especially in a case of a peripheral 
center that may lack or cannot afford

laparoscopy.

\section{REFERENCES}

1. Hamada Y, Takada K, Nakamura Y, Sato M, Kwon AH. Temporary umbilical loop colostomy for anorectal malformations. Pediatr Surg Int. 2012; 28:1133-6.
2. Cameron GS, Lau GYP. The umbilicus as a site for temporary colostomy in infants. J Pediatr Surg. $1982 ; 17: 362-4$.

3. Sauer CJE, Langer JC, Wales PW. The versatility of umbilical incision in the management of Hirschprungs disease. J Pediatr Surg. 2005; 40:385-9.

\section{Address for Correspondence*:}

Dr. Yogesh Kumar Sarin, Director Professor \& Head, Department of Pediatric Surgery,

Maulana Azad Medical College, New Delhi-110002

E mail: yksarin@hotmail.com

(c) 2015, Journal of Neonatal Surgery

Submitted: $15-03-2015$

Accepted: 22-03-2015

Conflict of interest: The author is editor of the journal but he is not involved in decision making of this manuscript.

Source of Support: Nil

How to cite: Raj P, Sarin YK. Umbilicus: a site for stoma in Hirschsprung's disease. J Neonat Surg. $2015 ; 4: 24$. 